\title{
Z. WÓYCICKI
}

Institut f. allg. Botanik der J. Piłsudski - Universität, Warszawa.

\section{ƯBER DIE HETEROMORPHIE ${ }^{1}$ ) DES GYNÄCEUMS BEI ROBINIA PSEUDACACIA L.}

\section{T E I L.}

Als ich im J. 1934 Material für meine Untersuchungen über die Ausscheidungsdrüsen in den Blüten von Robinia Pseudacacia sammelte, fiel mir an einer hinter dem Gebäude der früheren sogen. "Szkoła Główna” wachsenden Robinie die ungemein grosse Anzahl abnormer Gynäceen auf. Einzelne, normale Pistillen mit gut ausgebildetem Gynophorstiel ${ }^{2}$ ) und mehreren Samenanlagen im Fruchtknoten traten in den Blüten der erwähnten Robinie nur ausnahmsweise auf und nur diese Blüten brachten Hülsenfrüchte - von verschiedener allerdings Grösse - mit gut ausgebildeten und - wie ich mich teilweise selbst überzeugte - normal keimenden Samen hervor. Alle anderen Gynäceen fielen frühzeitig ab, ohne Früchte auszubilden.

Wie an anderer Stelle bemerkt ${ }^{3}$ ), erwähnt bereits D am-

1) Heteromorphie (M a sters) — „Unregelmässige Ausbildung eines Organes". Penzig O. 1921, Bd. 1, p. VI.

2) T a u bert, P. drückt sich so aus: „Fruchtknoten gestielt”. Die natürlichen Pflanzenfamilien v. Engler, A. u. Pran tl, K., III Bd., 3 Abt., p. 274.

Vele nowsky, J. (1910, p. 914) sagt darüber: „Es scheint da, als ob der Gynophorstiel wirklich der Blütenachse entspräche, denn nicht selten sind auch die Staubblätter an diesen Stiel angewachsen".

Schneider, C. K. bezeichnet den Gynophor als „stielartige Verlängerung der Blütenachse unter dem Gynäceum“. Handwörterbuch, Leipzig, 1905 , p. 25.

$\left.{ }^{8}\right)$ Wó y ci c ki, Z. 1937, „La Cellule“, Bd. XLV, Heft 3. 
mer, unter Berufung auf Göschke, in der von ihm selbst übertragenen „Teratology" von M a sters, dass bei Robinia Pseudacacia Blüten mit mehreren Pistillen auftreten. Nähere Einzelheiten über die gegenseitigen Beziehungen und den anatomischen Bau der einzelnen Bestandteile dieses zusammengesetzten Gynäceums sind bei Dammer allerdings nicht zu finden ${ }^{1}$ ).

Eine allgemeine Übersicht über den Charakter der in Frage kommenden Gynäceen bieten die Fig. 65, 66, 67, 68.

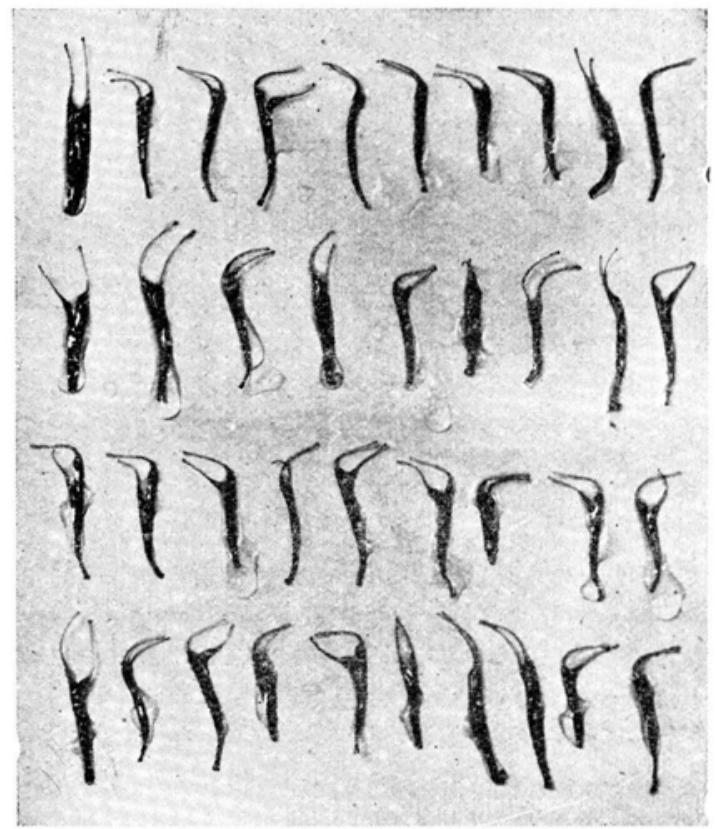

Fig. 65 .

Robinia Pseudacacia. Abnorme Gynäceen, nat. Gr.

In Fig. 65 sehen wir 38 Doppelgynäceen von verschiedenen gegenseitigen Beziehungen; es überwiegen jedoch dieje-

1) ,Bei den früher bereits erwähnten Fällen von Füllung der Blumen bei Robinia Pseudacacia monophylla beobachtete Göschke Beispiele mit mehreren Pistillen, von denen eins oder das andere petaloid geworden war" (L. c., p. 338). Ungefähr dasselbe, in allgemeinerer Form, wiederholt D a mmer im Kapitel über die „Polyphyllie des Gynäceums“: „Bei Papilionaceen, wo gewöhnlich nur ein Carpell entwickelt wird, findet man gelegentlich zwei oder mehr, wie bei Wistaria, Gleditschia, Trifolium, etc.“ (L. c., p. 417). 
nigen, in denen erst die Griffel beider Komponenten frei sind. Über den Charakter und Ausbildungsgrad der verwachsenen ${ }^{1}$ ) Karpelle informiert näher Fig. 66, in der die abnormen Gynäceen etwas mehr als zweifach vergrössert sind. Sowohl in der ersten (2) wie in der zweiten Reihe (11) sehen wir Pistillen, die sogar bis zum Griffelansatz seitlich miteinander verwachsen sind. Die Gynäceen 1, 7, 9, 14 setzen sich aus zwei nur an der Fruchtknotenbasis verwachsenen Karpellen zusammen (bei $\mathrm{Nr} 7$ etwas höher). Die Gynäceen 4, 5, 8 u. 9 stellen doppelblättrige Gebilde dar, in denen das schwächer entwickelte Karpell (ähnlich wie in 5 u. 8) stark deformirt ist. Die Pistillen der Gynäceen 3, 5, 6, 10, 12 u. 13 sind wie aus den gegeneinander gewendeten Griffeln deutlich ersichtlich (vgl. den histologischen Teil der vorliegenden Arbeit) - miteinander an den Bauchrändern verwachsen.

Wir hatten bisnun mit zweiblättrigen Gebilden zu tun, damit sind jedoch alle Fälle der Gynäceumstrukturanomalien bei der erwähnten Robinie nicht erschöpft, da ausser denselben auch Gynäceen vorkommen, die aus drei und sogar vier Karpellen zusammengesetzt sind (Fig. 67 B). Im letzten Falle sind zwei Karpelle $(1,2)$ ungefähr gleich stark entwickelt, das dritte (3) ist bereits viel schwächer ausgebildet, während das vierte den kleinsten Fruchtknoten hat und teilweise mitten zwischen den offenen Karpellhälften des dritten Fruchtblattes steckt. Der Griffel des vierten Karpells ist in der Mitte eingekrümmt

1) Es sei hier gleich bemerkt, das ich mich in dieser Arbeit, ähnlich wie in der vorhin zit., weiter an die Terminologie von Go ebel u. Troll halten werde. Mit Go e bel $(1898$, p. $45 ; 1901$, p. 723$)$ bezeichne ich als, ,kongenitale Verwachsung“ diejenigen Fälle, in denen die Karpelle vom Gynophor angefangen innig miteinander verwachsen sind, manchmal sogar ohne jede Spur eines Grenzgewebes oder einer Naht; diese treten erst an denjenigen Stellen zutage, wo die Spaltung der bisher ,kongenital“ verwachsenen Organe erfolgt. Von ,postgenitaler Verwachsung“ spreche ich - Troll folgend (1935, p. 29) - dann, wenn sich beispielsweise eine Bauchnaht bilde oder wenn nach der Spaltung der Karpelle bezw. der Karpellhäften an einer weiteren Stelle ihre Wiedervereinigung in der gleichen oder auch einer anderen Form erfolgt (z. B. wenn nach der Spaltung der linken Karpellhäfte des oberen von der rechten Karpellhälfte des unteren Karpells weiter ihre Wiederverwachsung bzw. Vereinigung der linken Karpellhälfte des oberen mit derjenigen des dominanten unteren Karpells erfolgt).

K üster (1925), wenn er von „Verwachsung“ (1. c., p. 35) spricht wendet die erwähnten Termine nicht an. 


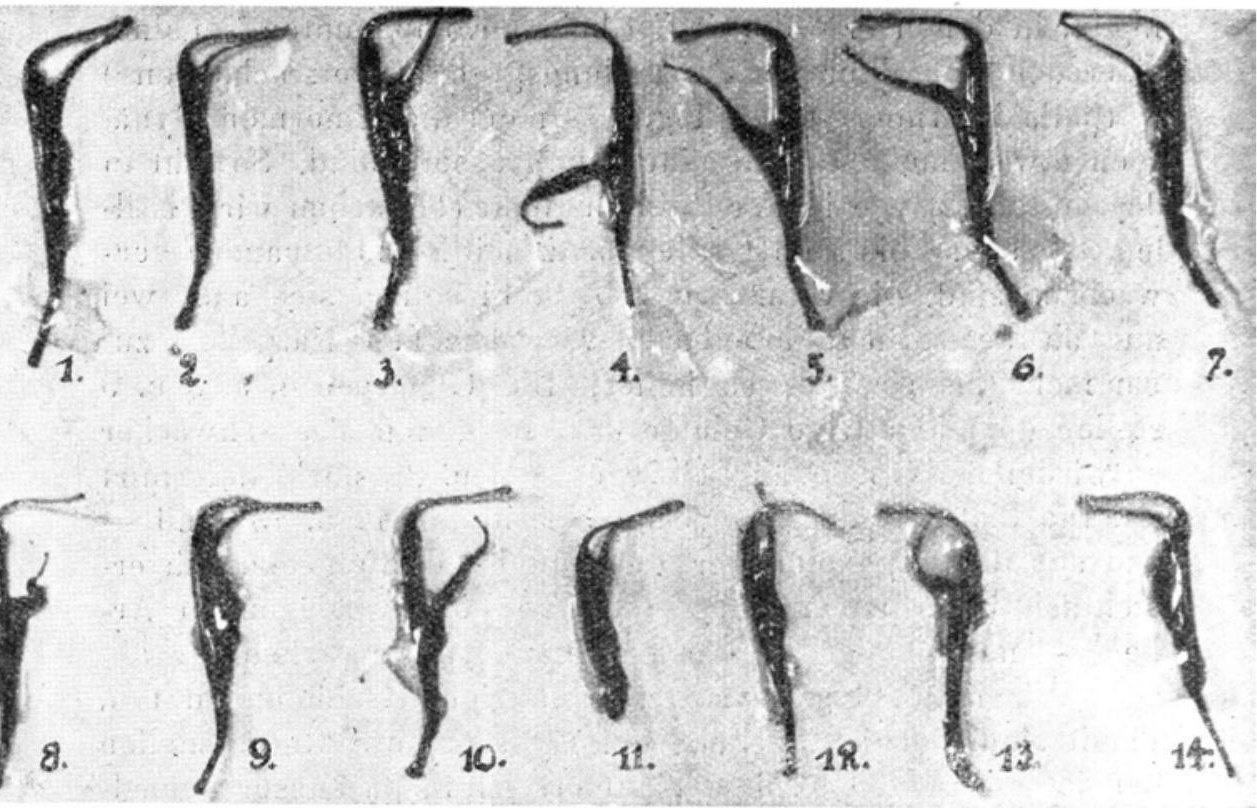

Fig. 66.

Robinia Pseudacacia. Zweikarpellige Gynäceen. Vergr. $>$ als $2 \times$.

und zwar derart, dass der Terminalteil mitsamt der Narbe in der oberen angeschwollenen Fruchtknotenpartie des dritten Karpells stecken bleibt ${ }^{1}$ ). Nach Auslaugung mit Javell'scher' Lauge zeigte es sich, dass diese Narbe noch schwächer als die in Fig. 68 B abgebildete entwickelt und mit sehr langen Haaren bewachsen war, während der Griffel, von der Narbe angefangen, eine offene Rinne bildete, deren Ränder unmittelbar in die Ränder der unverwachsenen Fruchtblatthälften übergingen.

Was die Narben anbelangt, sei gleich bemerkt, dass dieselben sehr verschieden ausgebildet sind. Manche, wie die in Fig. 67 A abgebildete oder die im Fruchtknoten $\mathrm{N} 4$ in Fig. 67 B steckende, sehen so wie die in Fig. $68 \mathrm{~A}$ abgebildete Narbe bzgw. sehr ähnlich aus und sind abgeplattet. Die Narben der Griffel 1 u. 2 in Fig. $67 \mathrm{~B}$ gehören dagegen zu derselben Ka-

1) Eine nähere Analyse der Vorwachsungsart und der Gewebebeziehungen solcher zusammengesetzten Karpellgruppen ist im II B Teil der Arbeit enthalten. 


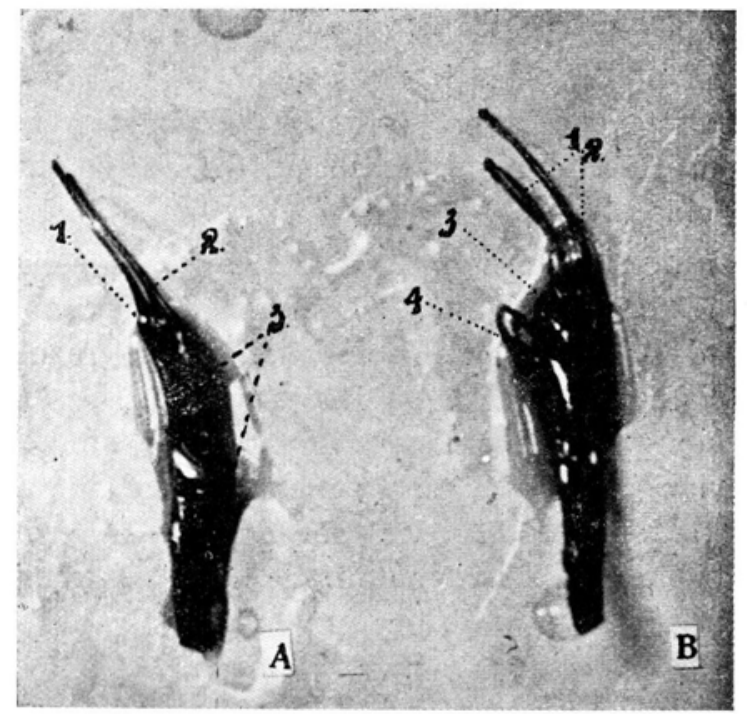

Fig. 67.

Robinia Pseudacacia. A-Dreiblättriges Gynäceum, $4 \times$ vergr. Zwei Karpelle (mit Griffeln) stärker entwickelt, das dritte griffellos und in der Entwicklung zurückgeblieben. B - Vierblättriges Gynäceum. Die Karpelle 1,2 und 3 besitzen normal ausgebildete Griffel u. Narben, das vierte mit den unverwachsenen Rändern besitzt einen eingekrümmten Griffel, der teilweise zwischen den offenen Karpellhälften des dritten Frachtblattes steckt.

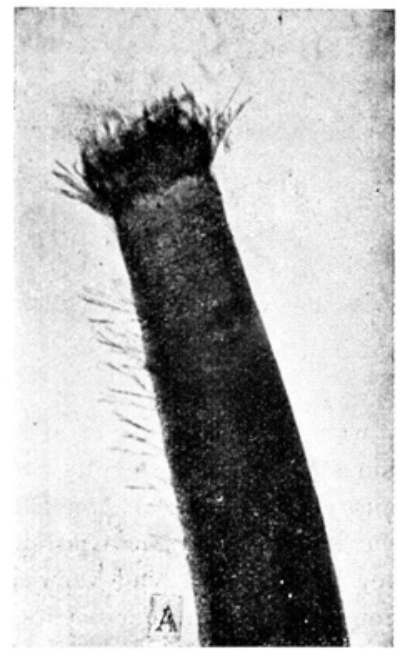

Fig. 68.

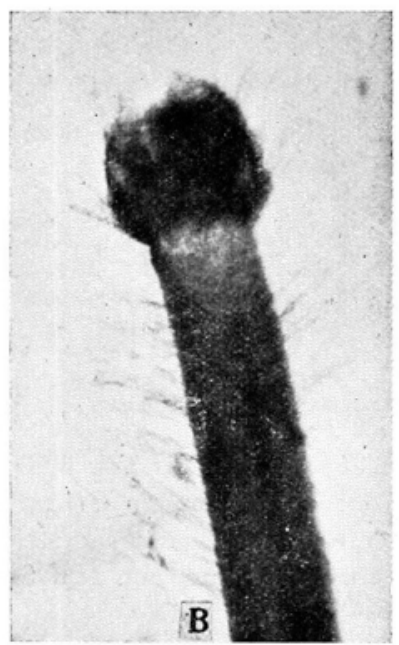

B

Robinia Pseudacacia. A-Griffel u. Narbe des in $67 \mathrm{~A}$ abgebildeten Gynäceums. B-Griffel (teilweise) u. Narbe der Karpelle 1 u. 2 in Fig. 67 B. Vergr. $20 \times$. 
tegorie wie die in $68 \mathrm{~B}$ abgebildete Narbe und sind mehr oder weniger oval und an der Basis stumpf. Die Basis ist ebenso wie der Griffel von allen Seiten gleichmässig mit langen, mehrzelligen Haaren bedeckt. Der in $68 \mathrm{~A}$ abgebildete Griffel ist nur an einer Seite behaart.

Ausser den eben dargestellten Heteromorphiefällen begegnete ich auf der erwähnten Robinie Gebilden, die durch A،lhäsion ${ }^{1}$ ), d. i. Verwachsung eines scheinbar einzelnen, bzgw. mehrerer Pistillen (Fig. 69 D) mit unvollständig entwickelten Staubgefässen oder Mittelformen entstanden sind. Sowohl in Fig. $69 \mathrm{~A}$ wie Fig. $69 \mathrm{~B}$ u. $69 \mathrm{C}$ sehen wir auf der Ventralseite der Fruchtknoten ungefähr in der Mitte Antheren auf Filamenten sitzen, die entweder auf der ganzen Länge mit den

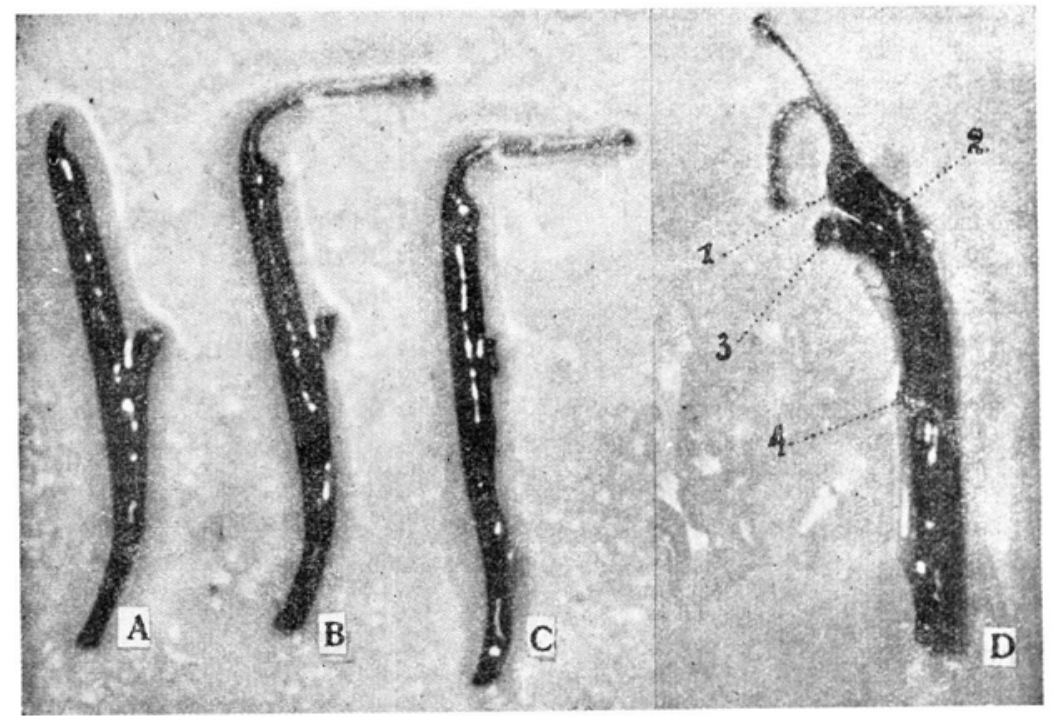

Fig. 69.

Robinia Pseudacacia. Adhäsion. In Fig. $69 \mathrm{~A}$ wurde der Griffel beim Präparieren abgebrochen. In Fig. $69 \mathrm{D}$ besass der am stärksten entwickelte Stempel einen stark gekrümmten Griffel mit einer unausgebildeten Narbe. Der Stempel N 2, kürzer als der vorherige, besitzt einen langen geraden Griffel. Das Zusatzorgan (3) war mit dem Stempel 1 in der Bauchnahtgegend verwachsen. Die nackte Samenanlage (N 4) steckt im offenen Fruchtknoten des Karpells N 2. Vergr. $4 \times$.

1) Vgl. D a m mer-M a sters, p. 49; auch Penzig, O, p. I. 
Geweben der Bauchnaht innig verwachse» (69 A u. 69 B) oder nur lose mit derselben vereinigt sind (69C).

i ie Gestalt des rinnenförmigen Staubfadens unterhalb der Antheren ist in Fig. 71, seine Beziehungen zur Bauchseite der unvollstäıdig verwachsenen Fruchtknotenwände in Fig. 70 dargestellt. Auf beiden Abbildungen sieht man überdies, dass die Fadenspitze über die Anthere hinausragt und eine Spiralrinne bildet, die mit cinem an normal ausgebildete Narben erinnerndem Köpfchen endet (Fig. 71 u. 72). Weiter sei noch erwähnt, dass in der Spiralrinne median ein schwach ausgebildetes Leitbündel läuft, das sich in einer gevissen Entfernung von der Spitze des dargestellten Gebildes verliert.

Das dargestellte Gebilde ist somit zur Kategorie der heterotopischen Erscheinungen hinzuzurechnen, mit a. W. als

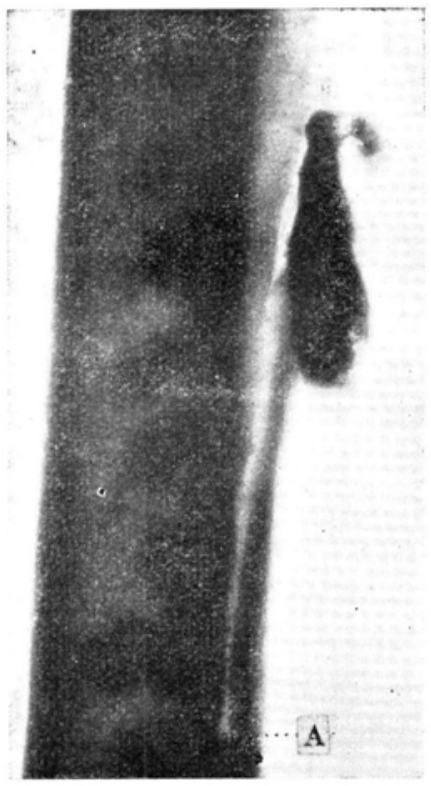

Fig. 70 .

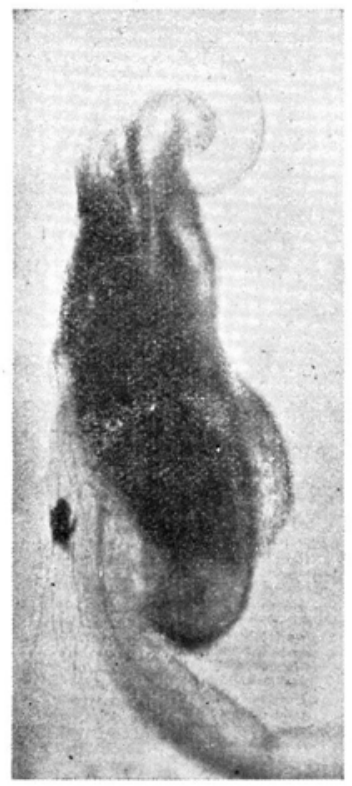

Fig. 71 .

Fig. 70. Robinia Pseudacacia. Verwachsung des Gynäceums mit einem unvollständig ausgebildeten u. deformirten Staubgefäss. Bei A ist die Verwachsung vollständig, weiter oben wird das Filament frei u. steckt teilweise im offenen Fruchtknoten. Vergr. $20 \times$.

Fig. 71. Robinia Pseudacacia. Der Staubfaden, die Anthere und die Fadenspitze der Fig. 70 in $50 \times$ Vergr. Der Faden ragt uber die Anthere hinaus und bildet über derselben einige Windungen. 
Grundtendenz seiner Entwicklung tritt die Tendenz zur Ausbildung eines Staubgefässes auf, an dessen Spitze sich jedoch

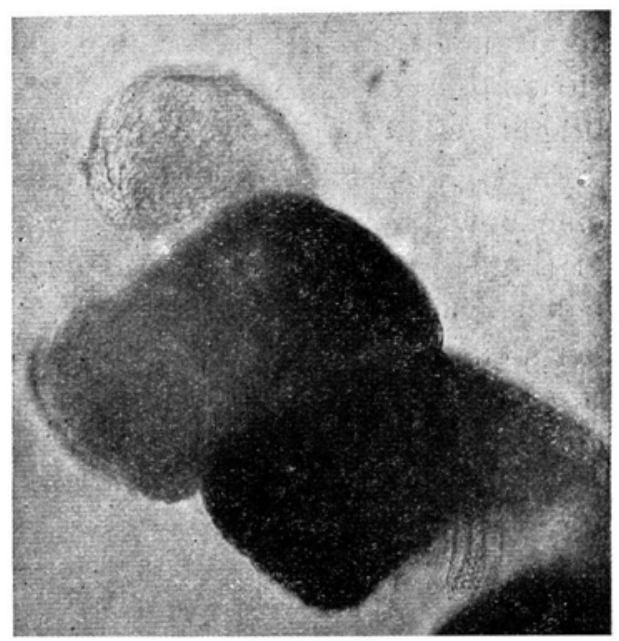

Fig. 72 .

Robinia Pseudacacia. Das über die Anthere hinausragende Zusatzorgan. Die Koppelfö:mige Spitze ist aus papillenartigen Zellen aufgebaut. In den unteren Windungen läuft median ein Leitbündel. $65 \times$.

Gewebe bilden, welche den Karpellen eigentümlich sind $\left.{ }^{1}\right)$. Es erinnert dies gewissermassen an die modifizierten Staubgefässe bei Papaser, die Mohl beobachtete, in denen an der Spitze der Anthere.... schon Narbenpapillen entstanden ${ }^{2} \%$. Die Antherenwände der in Fig. 69,70 u. $7 \mathrm{I}$ abgebildeten Staubgefässe wie auch des Gebildes, dessen Spitze Fig. 72 darstellt, enthalten typische $\mathrm{Hy}$ podermzellen nit glockenartigen Leisten (Fig. 73). Im Innern der Pollensäcke liegen Pollenkörner von normaler Gestalt (Fig. 74), manche von ihnen haben bereits Pollenschläuche ausgetrieben (Fig. 75).

Als Beispiel eines Fruchtknotens, dessen Wände unvollständig verwachsen sind, möge Fig. 76 dienen. Wir sehen hier in $20 \times$ Vergrösserung ein aus einem Karpell u. einem stark deformierten Staubgefäss zusammengesetztes Gebilde (Fig. 76 B). Die Ränder des breitgeöffneten Fruchtknotens sind nach innen eingebogen. Samenanlagen sind nur einige vorhanden. Drei sind auf der Abbildung sichtbar (Fig. $76 \mathrm{~A}, 1,2,3$ ). Alle Samenanlagen sind abnorm ausgebildet, zwei (Fig. $76 \mathrm{~A}$, 1, 2) stellen sehr eigenartige Bildungen vor (Fig. 77). Ihr Zentralkörper wird von einer aus grossen Parenchymzellen aufgebauten Kugel gebildet, die von 4 an der Basis breiteren

1) K üs te r, E., 1916, p. $313-315 ; 1925$, p. 246.

$\left.{ }^{2}\right)$ G o e bel, K., S che n k's Handbuch der Botanik, Bd. III, p. 123, Fig. 7. 
und gegen die Spitze sich plötzlich stark einschmälernden Lappen umfasst wird (Fig. $77 \mathrm{~A}$ ). Zwei Lappen liegen nach aussen, die zwei anderen, kleineren, liegen unter ihnen (Fig. 77 B). Die dargestellten Gebilde erinnern als typische „Missbildungen” sehr stark an die „vergrünten Samenanlagen" ${ }^{1}$ ), die Celakov$\mathrm{sky}$ bei Hesperis matronalis beobachtete.

Was das Organ anbelangt, das in Gestalt eines ziemlich langen, stellenweise eingeschnürten Sackes am Eingange zum breitgeöffneten Fruchtknoten steht, so kommt sein gemischter Charakter sowohl im Bau der Wände, die genau so aussehen wie in der früher dargestellten Theka (Fig. 73), wie auch in der Struktur des Filaments und des spiralen,

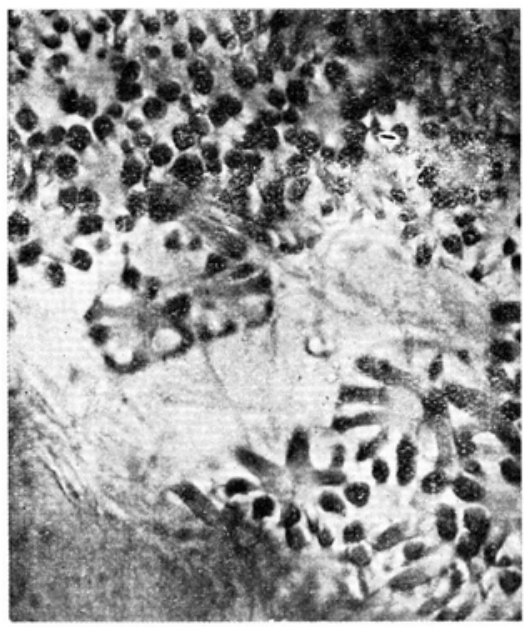

Fig. 73.

Robinia Pseudacacia. Hypodermzellen in den Antheren der in Fig. 69, 70 u. 71 abgebildeten Staubgefässe. Nach einem zerzupften Präparat photogr. $640 \times$.

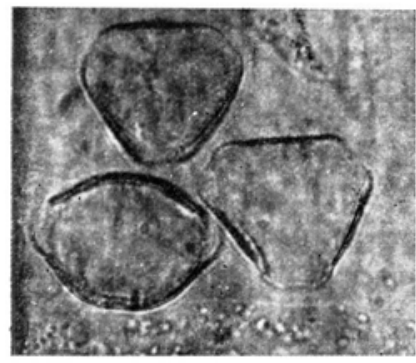

Fig. 74.

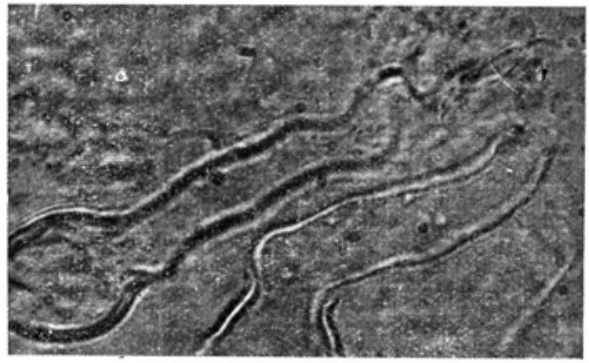

Fig. 75 .

Fig. 74 u. 75. Robinia Pseudacacia. Pollenkörner u. Pollenschläuche aus den Pollensäcken der in Fig. 69, 70 u. 71 abgebildeten Staubgefässe. Nach einem zerzupften Präparat photogr. $640 \times$.

1) G o e bel (1913) unterstreicht in Bezug auf dieselben, dass ,....in allen Fällen der Vergrünung (diese) begleitet wird von einer Verkümmerung des Nucellus" (1. c., p. 331). 
über den Sack hinaısragenden Fortsatzes zum Ausdruck. Der breite rinnenförmige Faden dieses Organs besass ca 6 typische

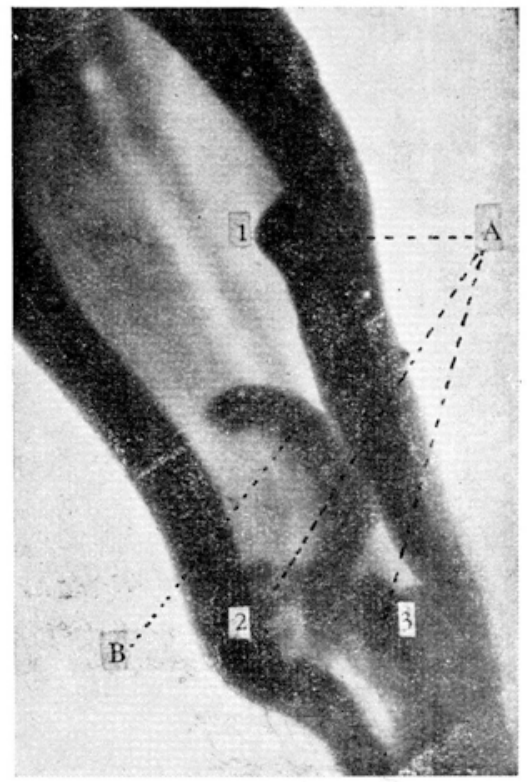

Fig. 76 .

Robinia Pseudacacia. Teil des unverwachsenen Fruchtknotens mit einem degenerierten Staubgefäss am Eingang. A - drei Samenanlagen, deren Bau Fig. 77 darstellt; B - das Gebilde, das durch den Bau der Wände an eine

Theka erinnert. $20 \times$.
Spaltöffnungsapparate; dorsal läuft eine Reihe von Zellen, die Hemitropien des Kalkoxalates enthalten ${ }^{1}$.

Die erwähnten Zellen laufen in der Regel streifenartig längs der Karpellhälften normaler Karpelle ${ }^{2}$ ). Die Kristalle füllen manchmal die Zellen auf diese Weise aus, dass sie sich mit ihren flachen (in Fig. 78 deutlich sichtbaren) Enden an die Zellwand anlehnen. Ob und in welchem Grade sie ,...in Verdickungen der Zellwand eingebettet sind...", kann ich nicht entscheiden, da ich eher geneigt bin zu vermuten, dass als "Verdickungen der Zellwand" jene flachen Endungeı angenomnen wurden, die iı polarisierten Lichte viel weniger deutlich als im gewöhulichen Lichte auftreten.

Es sei hier hervorgehoben, dass in den Pollensäcken der Theken die mit den über die Antheren hinausragenden Spiralfortsätzen versehen sind, die keimenden Pollenkörner (Fig. 75) nicht nur Pollenschläuche in der Richtung zur papillösen Spitze austreiben, sondern auch einen deutlichen Weg besitzen, längs dessen sie sich dorthin richten. Er führt über das Gewebe, das die erwähnten Kalkoxalathemitıopien in grösserer Anzahl enthält. Was die Pollenkörner anbelangt,

1) Solereder, K., 1899 p. 302.

$\left.{ }^{2}\right)$ Nach Solerede r: ,gibt... solche mit stäbchenförmigen Krystallen erfüllte Pallisadengewebezellen... W e y l a nd (1893) unter den Galegeen für... Robinia..." an (1. c., p. 302). 
so haben sie - in dem erwähnten Falle - einen sehr verschiedenen Umriss. Ausser den in Fig. 74 u. 75 abgebildeten

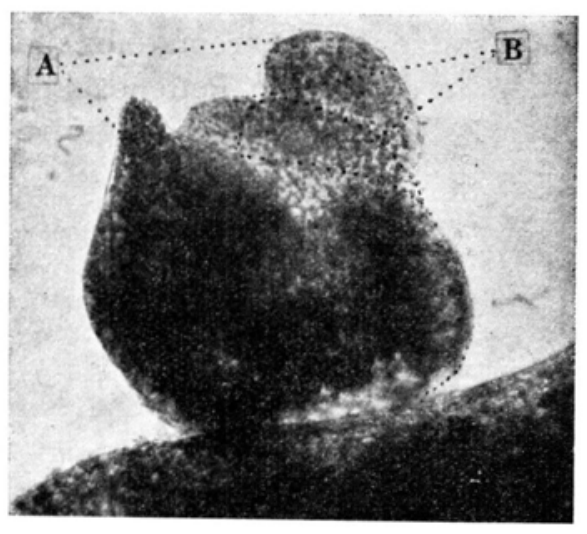

Fig. 77 .

Robinia Pseudacacia. Abnorm ausgebildete Samenanlage $\left(A_{2}\right.$ aus Fig. 76). A-Aussenlappen, B-Innenlappen. $65 \times$.

treten auch grössere und kleinere Köıner auf. Fast alle besitzen dickere als sonst und nicht selten stark gefaltete Membranen, die meistens keine Austrittstellen und auch keine für die Exine eigenartige Struktur besitzen (Fig. 79).

Verhältnissmässig nicht lange vorher (1935) beschrieb ähnliche Missbildungen bei Zelkowa crenata $\mathrm{F}$ inn. F i n $\mathrm{n}$ vermutet, dass sie mit der "Nacktsamigkeit” der erwähnten Pflanze zusammenhängen ${ }^{1}$ ). Ob dieser Zusammenhang wirklich den Tatsachen entspricht und einen allgemeinen Charakter besitzt, kann ich nicht feststellen, da bei Robinia auf sehr vielen nackt. samigen Fruchtknoten keine Spur von irgendwelchen, den Theken eigentümlichen (ieweben, zu sehen war. Überdies treten die Missbildungen bei Robinia an einer anderen Stelle als bei Zelkowa auf. Bei Zelkowa entwickeln sich die Antheren - wie Fin n feststellte - gewöhnlich in dem oberen Teil des Fruchtknotens, in der Nähe der Narben. Bei Robinia dagegen

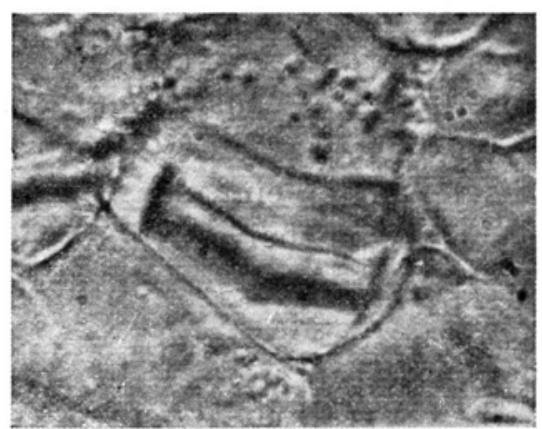

Fig. 78.

Robinia Pseudacacia. Kalkoxalathemitropien in den Dorsalzellen des in Fig. 76 mit $B$ bezeichneten Organs. $1020 \times$.

1) „Die ,Nacktsamigkeit" hat häufig eine Bildung der Antheren auf dem Fruchtknoten zur Folge“ (1. c., p. 182). 
traten die erwähnten Bildungen - wie aus Fig. $69 \mathrm{~A}, \mathrm{~B}$ u. C ersichtlich - ungefähr auf halber Höhe des Gynäceums und nur ausnahmsweise [in den komplizierteren Verwachsungen (Fig. 69 D)] näher der Fruchtknotenspitze auf. Ubereinstimmend mit Fin n dagegen ist der Pollen in derartigen Pollensäcken auch bei Robinia, ebenso wie bei Zelkowa, stark degeneriert.
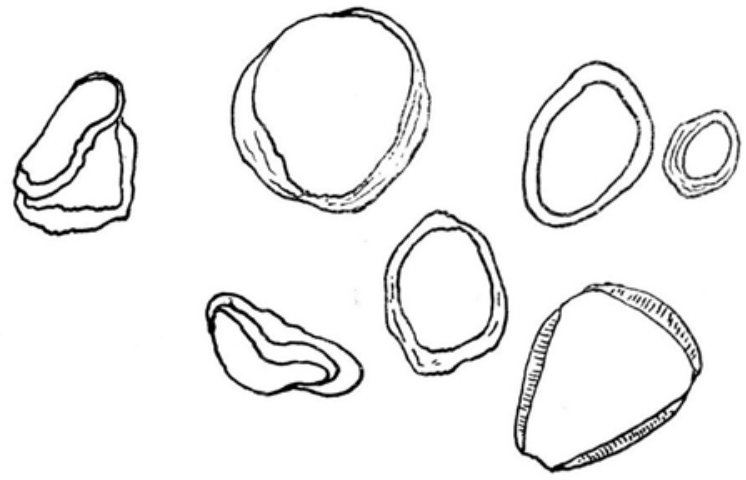

Fig. 79 .

Robinia Pseudacacia. Umriss u. Charakter der Membran in den Mikro- u. Makropollenkörnern die aus den Pollensäcken der in Fig. 69, 70, 71 u. 76 B abgebildeten Theken stammen. $620 \times$.

Was die Ursachen derartiger Verwandlungen anbelangt, so sei an die gedankenreichen und überaus treffenden Worte von Goebel aus dem J. 1898 erinnert: "Es werden also, je verwickelter ein Organkomplex der Pflanze ist, desto leichter Missbildungen eintreten können, denn schon kleine Störungen genügen, um bei dem Einwandern der organbildenden Stoffe in die Anlagen Änderungen hervorzurufen.... Wenn z. B. éinige Moleküle solcher Substanzen, welche die Antherenbildung anregen, nur um $1 / 1000 \mathrm{~mm}$ rechts oder links vom Wege abirren oder sich auf ihrer Wanderung in den Blütenvegetationspunkt verspäten oder verfrühen, so werden an einem Carpellblatt oder einem Blumenblatt Antherencharaktere teilweise auftreten" ${ }^{1}$ ).

Zum Schluss möchte ich die Frage des Auftretens nackter Samenanlagen bei den Angiospermen berühren. B a r a n ov

1) Go e b e l, K., 1898, p. 174 
(1927), der nackte Samenanlagen bei Vitis (Rasse Mouzvédre) becbachtete, glaubt, dass dies „eine Folge der Entwicklung einer grossen Anzahl von Samenanlagen” sei ,...diese Samenanlagen üben auf die Fruchtknotenwände einen Druck aus, rufen einen Riss hervor an der Stelle, wo der Widerstand am geringsten ist, nämlich im Gewebe des Griffels" ${ }^{1}$ ).

F in n $(1935)^{2}$ ) vertritt die Meinung, das dies nicht als normale Erscheinung betrachtet werden kann im Sinne B aranows - da wir hier ,mit einer wesentlichen Störung im Bau einer der charakteristischen Grundstrukturen der Angiospermen" zu tun haben ${ }^{3}$ ). Zu den Ursachen der teratologischen Nacktsamigkeit bei Zelkowa crenata übergehend, glaubt Finn, dass als eine derselben wenigstens die Infektion des Fruchtknotens durch eine näher nicht bekannte Insektenlarve anzusehen ist. Die spezifischen Ausscheidungsprodukte dieser Larve rufen ,eine Veränderung in den Korrelationen zwischen den Teilen der Blüte hervor" ${ }^{4}$ ). Bei Robinia Pseudacacia sind alle Nacktsamigkeitsfälle zweifellos eine Folge der ungenügenden Entwicklung der Karpelle, die durch die Veränderung der Korrelationsbeziehungen zwischen den benachbarten Organen hervorgerufen wird. So können z. B. zwei Karpellanlagen miteinander ab initio (kongenital) ${ }^{5}$ ) auf der ganzen Grenzfläche verwachsen (s. Wó y c i cki, 1937, Abb. A). In dem Karpell, das mit dem Rücken gegen das Schiffchen gewendet ist und in diesem Falle sichtlich über die oberen Karpelle dominiert, sind die Karpellhälften am Rande mittels einer deutlichen Bauchnaht verwachsen; die linke, übermässig entwickelte Karpellhälfte des dominanten Karpells, steht jedoch in inniger Verbindung mit der linken Karpelhälfte des stark recessiven, oberen (mit dem Rücken gegen die Fahne gewendeten) Karpells. Auf diesem, offenen Karpell liegt gleich über der Placenta der linken Karpellhälfte eine Samenanlage, die infolge der unvollständigen Entwicklung der rechten Karpellhälfte nackt geblieben ist. Nackte Samenanlagen bei Robinia haben

1) L. c., p. 110.

$\left.{ }^{2}\right)$ Siehe die durch diesen Autor angeführte Lit. über die ,Nacktsamigkeit'.

3) F in n W., l. c., p. 184.

4) ", 1. c., p. 185.

5) Go ebel K., 1898, p. 45 u. $723-725$. 
wir auch in dem in Fig. 80 dargestellten Falle. Hier sind die Karpelle nur an der Basis des Gynäceums verwachsen. Weiter oben ist das untere, mit dem Rücken gegen das Schiffchen zugewendete Karpell normal entwickelt, seine Hälften verwachsen am Rande zu einer deutlichen Bauchnaht; in dem oberen Karpell dagegen bleiben beide Karpellhälften in der Entwicklung zurück und schliessen nicht zusammen, so dass die Samenanlagen infolgedessen nackt bleiben.

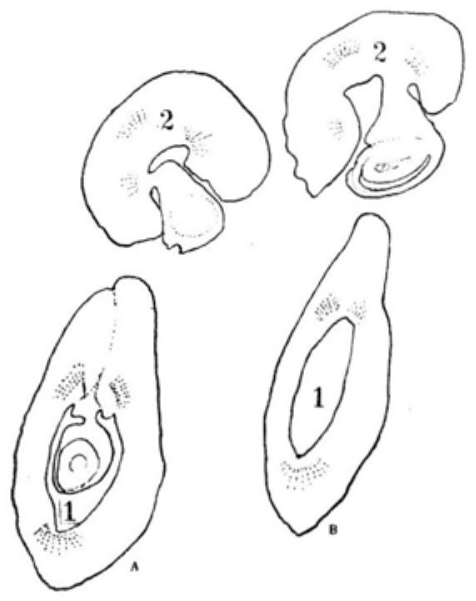

Fig. 80 .

Robinia Pseudacacia. A-Schema der Verhältnisse an der Stelle, wo die Karpelle bereits frei sind. Das untere Karpell mit einem normalen Fruchtknoten, das obere mit unvollständig entwickelten Karpellhälften u. nackten Samenanlagen. B-Schema der Verhältnisse an der Übergangstelle des Fruchtknotens des unteren Karpells in den Griffel (vgl. das

Gynäceum No 6 in Fig. 66).

Aus Fig. 80 ist weiter ersichtlich, dass die Entwicklung der erwähnten Karpelle nicht paralell zueinander verläuft, da auf der Ebene, in welcher der geschlossene Fruchtknoten keine Samenanlagen enthält (Fig. 80, B, 1), die Samenanlage in dem in der Entwicklung zurückgebliebenen Karpell gerade am reichlichsten ist, wenn auch abnorm, ausgebildet (Fig. 80, B, 2). Eine davon, u. zw. die in Fig. $80 \mathrm{~B}$ abgebildete, entspricht der Fig. 81 meiner Publikation aus dem I. J.

Die Fig. 81 u. 82 stellen Fälle vor, in denen beide Karpelle nackte Samenanlagen aufweisen. Sie treten hier in der Unterpartie der erwähnten mit den linken Karpellhälften verwachsenen Organe (in der Nähe des Gynophors) auf (Fig. 81 A). Weiter oben beginnen die Karpelle zu verwachsen (Fig. 81 B), um in der Nähe des Griffels schliesslich ganz frei zu werden (Fig. 81 G). Auch hier sind - ebenso wie im vorherigen Falle - die Karpelle ungleich entwickelt. Das mit dem Rücken gegen das Schiffchen gewendete untere Karpell ist stärker als das obere, über ihm gelegene, ausgebildet.

Viel seltener, beinahe ausnahmsweise, kommt es vor, dass in der Entwicklung beider benachbarten Organe ein sehr 
kleiner (Fig. 82) oder gar kein Unterschied vorhanden ist. Fig. 82 zeigt eine, sozusagen, relative Nacktsamigkeit, die erst auf Schnitten zutagetritl; hier nämlich stehen die Samenanlagen nicht am Rande der offenen Karpellhälften, sondern stecken im Innern der Fruchtknotenhöhlung, zu der ein schmaler Spalt zwischen den unverwachsenen Karpellhälften führt.

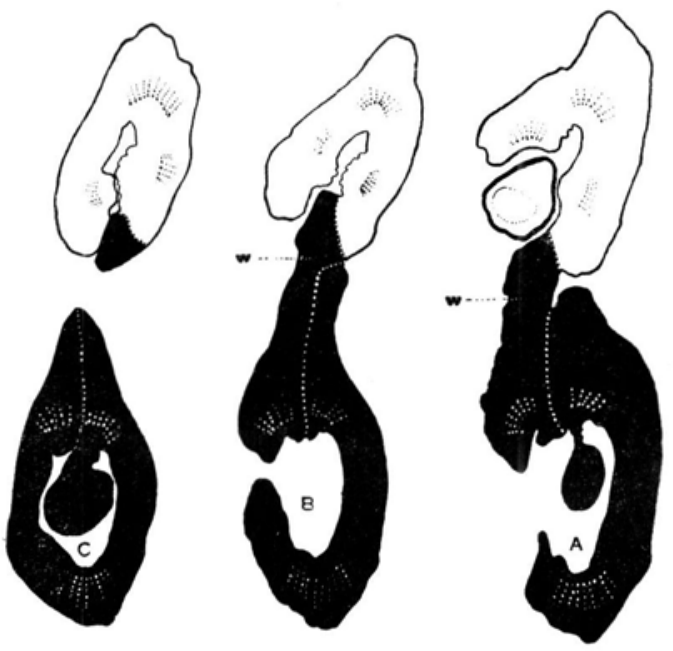

Fig. 81. A, B, C.

Robinia Pseudacacia. Schema der Verhältnisse in drei aufeinander folgenden Schnitten durch beide Karpelle. A, B - im unteren Karpell ist die linke Hälfte deformiert, im oberen die rechte schwach entwickelt. C - das untere Karpell besitzt einen geschlossenen Fruchtknoten, das obere bleibt weiter offen (vgl. das dritte Gynäceum in der zweiten Reihe im Fig. 65 oder das Gynäceum No 1 in Fig. 66). Bei W erfolgt die Spaltung der linken Karpellhälften beider Karpelle.

Aus der vorliegenden Übersicht könnte es scheinen, dass. bei unserer Robinia Nacktsamigkeit in denjenigen Fällen auf. tritt, wo zwei Karpelle vorhanden sind. Dies ist jedoch nicht der Fall, da, wie Fig. 83 zeigt, Nacktsamigkeit auch da vorkommt, wo drei in verschiedenen Beziehungen zueinander stehende Fruchtblätter auftreten (vgl. den II Teil).

Die in diesem Falle zur Gynäceumgruppe gehörenden Karpelle sind nur in der Nähe des Gynophors vereinigt. Schon auf der Höhe A (Fig. 83) ist das gegen das Schiffchen gewendete Karpell nicht nur frei, sondern seine Hälften sind 
miteinander am Rande innig mittels einer Bauchnaht verwachsen ${ }^{1}$ ).

Die beiden übrigen Karpelle (von denen eines gegen die Fahne, das zweite gegen den linken Flügel gewendet ist) bleiben in inniger Verbindung sowohl in der Rücken $^{2}$ ) - wie in der Bauchgegend. In der Nähe der Griffel endlich werden die durch spezifische Gewebeformen umgrenzten ${ }^{3}$ ) Karpellränder frei, so dass zwischen ihnen ein

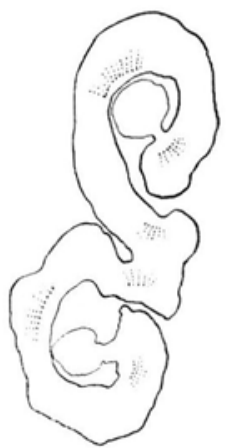

Fig. 82 .
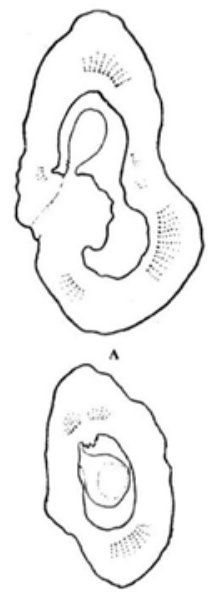
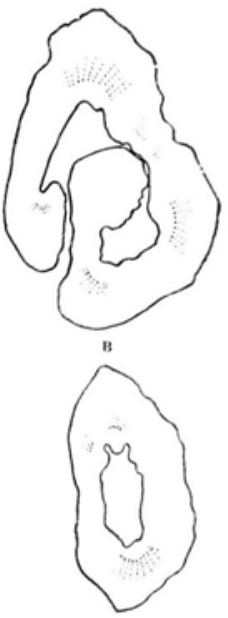

Fig. 83 .

Fig. 82. Robinia Pseudacacia. Schema der partiellen Nacktsamigkeit; die Samenanlagen liegen im Innern der Fruchtknotenhöhlungen, in welche Spalte zwischen den unverwachsenen Karpellhälften münden (vgl. Fig. 66, Gynäceum No 11).

Fig. 83. Robinia Pseudacacia. A u. B. Schema der Verhältnisse in einem aus drei verschieden ausgebildeten Karpellen zusammengesetzten Gynäceum.

anfangs noch schmaler, dann sukzessiv sich ausbreitender Spalt entsteht, in dem eine hypertrophisch ausgebildete und deformierte Samenanlage auf einem überaus stark entwickelten Funikulus sitzt.

1) Genaueres über die ungefähr auf der ganzen Länge herrschenden Verhältnisse in derartigen, bald verwachsenen, bald wieder freien Karpellen dreiblättiger Gynäceen siehe T. II. B.

$\left.{ }^{2}\right)$ Hier haben wir natürlich eine kongenitale „parakarpe“ (G o e b e l, 1900, p. 734) Verwachsung vor uns.

3) S. den II Teil dieser Arbeit [A u. B]. 
B a r a n o v, P. 1927. Ber. Deutsch. Bot. Ges., Bd. 45 .

D a m m e r, U. 1886. Pflanzen-Teratologie (von Maxwell T. Masters). Leipzig. Engler, A. und Pran tl, K. 1894. Die natürlichen Pflanzenfamilien, Bd. III, Abt. 3 .

F i n n, W. 1935. Ber. Deutsch. Bot. Ges., Bd. 53. H. 2.

G o e bel, K. 1883. Vergleichende Entwicklungsgeschichte der Pflanzenorgane. Schenk's Handbuch der Botanik, Bd. III. Berlin.

- 1898. Organographie der Pflanzen. I Teil. Jena.

- 1901. Organographie der Pflanzen. II Teil. Jena.

- 1913. Organographie der Pflanzen. I Teil. II Auflage. Jena.

K üster, E. 1916-1925. Pathologische Pflanzenanatomie. Jena.

P e n zi g, O. 1921-1922. Pflanzen-Teratologie. Berlin.

S c h n e i d e r, C. K. 1905. Illustriertes Handwörterbuch der Botanik. Leipzig. S o l e reder, H. 1899. Systematische Anatomie der Dicotyledonen. Stuttgart. Troll, W. 1935. Vergleichende Morphologie der höheren Pflanzen. Berlin. Velenowsky, J. 1905-1913. Vergleichende Morphologie der Pflanzen. Prag.

W ó y c i c ki, Z. 1937. La Cellule, Bd. 45, H. 5 . 